\title{
TAARI Modulates Cortical Glutamate NMDA Receptor Function
}

\author{
Stefano Espinoza', Gabriele Lignani', Lucia Caffino², Silvia Maggi', Ilya Sukhanov',3, Damiana Leo', \\ Liudmila Mus', Marco Emanuele', Giuseppe Ronzitti', Anja Harmeier', Lucian Medrihan', \\ Tatyana D Sotnikova',5, Evelina Chieregatti', Marius C Hoener', Fabio Benfenati', Valter Tucci', \\ Fabio Fumagalli ${ }^{2}$ and Raul R Gainetdinov*, ${ }^{*, 5,6}$
}

'Department of Neuroscience and Brain Technologies, Istituto Italiano di Tecnologia, Genova, Italy; '2Dipartimento di Scienze Farmacologiche e Biomolecolari, Università degli Studi di Milano, Milan, Italy; ${ }^{3}$ Department of Pharmacology, St Petersburg State Medical University, Petersburg, Russia; ${ }^{4}$ Neuroscience, Ophthalmology and Rare Diseases Discovery and Translational Area, pRED, Roche Innovation Center Basel, F. HoffmannLa Roche Ltd, Basel, Switzerland; ${ }^{5}$ Institute of Translational Biomedicine, Faculty of Biology, St Petersburg State University, St Petersburg, Russia; ${ }^{6}$ Skolkovo Institute of Science and Technology (Skoltech) Skolkovo, Moscow region, Russia

\begin{abstract}
Trace Amine-Associated Receptor I (TAARI) is a G protein-coupled receptor expressed in the mammalian brain and known to influence subcortical monoaminergic transmission. Monoamines, such as dopamine, also play an important role within the prefrontal cortex (PFC) circuitry, which is critically involved in high-o5rder cognitive processes. TAARI-selective ligands have shown potential antipsychotic, antidepressant, and pro-cognitive effects in experimental animal models; however, it remains unclear whether TAARI can affect PFCrelated processes and functions. In this study, we document a distinct pattern of expression of TAARI in the PFC, as well as altered subunit composition and deficient functionality of the glutamate $\mathrm{N}$-methyl-D-aspartate (NMDA) receptors in the pyramidal neurons of layer $\mathrm{V}$ of PFC in mice lacking TAARI. The dysregulated cortical glutamate transmission in TAARI-KO mice was associated with aberrant behaviors in several tests, indicating a perseverative and impulsive phenotype of mutants. Conversely, pharmacological activation of TAARI with selective agonists reduced premature impulsive responses observed in the fixed-interval conditioning schedule in normal mice. Our study indicates that TAARI plays an important role in the modulation of NMDA receptor-mediated glutamate transmission in the PFC and related functions. Furthermore, these data suggest that the development of TAARI-based drugs could provide a novel therapeutic approach for the treatment of disorders related to aberrant cortical functions.

Neuropsychopharmacology (20I5) 40, 2217-2227; doi: 10.1038/npp.20I5.65; published online I April 20I5
\end{abstract}

\section{INTRODUCTION}

Trace Amine-Associated Receptor 1 (TAAR1) is a G proteincoupled receptor found in several regions of the mammalian brain with the highest expression observed in the subcortical monoaminergic nuclei, such as the ventral tegmental area and the dorsal raphe, whereas low levels are detected in the striatum and several other brain areas (Borowsky et al, 2001; Bunzow et al, 2001; Lindemann et al, 2008; Di Cara et al, 2011). Recently, TAAR1 functions have been extensively characterized, with particular attention paid to its role in the modulation of the midbrain dopamine system and its possible implications in psychiatric diseases (Borowsky et al, 2001; Bunzow et al, 2001; Lindemann and Hoener, 2005; Sotnikova et al, 2009; Leo et al, 2014; Sukhanov et al, 2014). TAAR1 knockout (TAAR1-KO) mice display a higher

\footnotetext{
*Correspondence: Professor R Gainetdinov, Neuroscience and Brain Technologies Department, Istituto Italiano di Tecnologia, Via Morego, 30, Genoa 16163, Italy, Tel: +39010 7178 1516,

E-mail: raul.gainetdinov@iit.it

Received 21 October 2014; revised 21 February 2015; accepted 25 February 2015; accepted article preview online 9 March 2015
}

sensitivity to amphetamine and other psychostimulants (Wolinsky et al, 2007; Lindemann et al, 2008; Di Cara et al, 2011), and seem to have a supersensitive dopaminergic system (Wolinsky et al, 2007; Lindemann et al, 2008), making them an interesting model relevant for schizophrenia (Wolinsky et al, 2007). Accordingly, both full and partial TAAR1-selective agonists are efficacious in experimental rodent models of psychosis, such as pharmacologic or genetic mouse models of hyperdopaminergia, further supporting the idea that TAAR1 could represent a novel target for psychiatric diseases (Sotnikova et al, 2009; Espinoza and Gainetdinov, 2014). Although there is evidence suggesting a potential TAAR1 involvement in cognitive functions (Revel et al, 2013), no studies evaluated TAAR1 role in cortical regions so far. Recently, it has been shown that TAAR1 agonists were able to suppress hyperactivity in pharmacologic or genetic mouse models of glutamate NMDA receptor deficiency and improved cognition in rats treated with the glutamate NMDA receptor antagonist phencyclidine (Revel et al, 2013). Intriguingly also, TAAR1 agonists, tested in phMRI studies in mice, shared a similar pattern of brain activation with the antipsychotic olanzapine 
with the most prominent activation being observed in the medial PFC (Revel et al, 2013).

Thus, the aim of this study was to investigate the role of TAAR1 in the prefrontal cortex (PFC), a brain region important for cognitive functions. In particular, we explored whether TAAR1 might modulate cortical glutamatergic transmission as its deficiency is implicated in the pathophysiology of schizophrenia (Millan et al, 2012), which postulates, among other theories, involvement of NMDA receptor hypofunction (Mohn et al, 1999).

\section{MATERIALS AND METHODS}

\section{Animals}

TAAR1 knockout (TAAR1-KO) mice of mixed C57BL/ $6 \mathrm{~J} \times 129 \mathrm{~Sv} / \mathrm{J}$ backgrounds were generated as previously described (Wolinsky et al, 2007). Animal care and treatments were performed in accordance with the Guide for the Care and Use of Laboratory Animals (USA National Institutes of Health publication \#865-23, Bethesda, MD), and the protocols were approved by the Italian Ministry of Health. All TAAR1-KO and wild-type (WT) littermates were obtained from heterozygous mating, and genotyping was performed in all individuals. Mice of 8-16 weeks of age and of both sexes were used in all experiments. Mice were sex- and agematched in all experiments.

\section{Western Blot}

The crude synaptosomal fraction was obtained from the PFC extracts to evaluate glutamatergic receptor expression and composition (Fumagalli et al, 2012). The PFC was homogenized in a glass-glass potter using a cold buffer containing $0.32 \mathrm{M}$ sucrose, $1 \mathrm{mM}$ Hepes solution, $0.1 \mathrm{mM}$ EGTA, and $0.1 \mathrm{mM}$ PMSF, $\mathrm{pH}=7.4$, in the presence of a complete set of protease inhibitors (Sigma-Aldrich, Milan, Italy) and a phosphatase inhibitor cocktail (Sigma-Aldrich). The homogenized tissues were centrifuged at $1000 \mathrm{~g}$ for $10 \mathrm{~min}$; the resulting supernatant was centrifuged at $9000 \mathrm{~g}$ for $15 \mathrm{~min}$ to obtain the pellet corresponding to the crude synaptosomal fraction, which was resuspended in a buffer containing $20 \mathrm{mM}$ Hepes, $0.1 \mathrm{mM}$ DTT, and $0.1 \mathrm{mM}$ EGTA in the presence of a complete set of protease inhibitors and a phosphatase inhibitor cocktail. Ten micrograms of proteins for each sample were run on a SDS- $10 \%$ polyacrylamide gel under reducing conditions and then electrophoretically transferred onto nitrocellulose membranes (GE Healthcare, Milan, Italy). Blots were incubated with antibodies against the phosphorylated forms of the proteins and actin was used as loading control. Immunocomplexes were visualized by chemiluminescence using the Chemidoc MP Imaging System (Bio-Rad Laboratories).

\section{Real Time PCR}

Real-time PCR was performed as described in SI Materials and Methods.

\section{Electrophysiology Recordings}

Preparation of slices. For all patch-clamp experiments, we used 3-5-week-old wild-type and TAAR1-KO mice. Coronal slices containing the mPFC ( $350 \mu \mathrm{m}$ thickness) were cut using a Microm HM $650 \mathrm{~V}$ microtome provided with a Microm CU 65 cooling unit (both Thermo Fisher Scientific, Waltham, MA, USA). Slices were cut at $2{ }^{\circ} \mathrm{C}$ in a solution containing (mM): $87 \mathrm{NaCl}, 25 \mathrm{NaHCO}_{3}, 2.5 \mathrm{KCl}, 1.25 \mathrm{NaH}_{2} \mathrm{PO}_{4}, 0.5 \mathrm{CaCl}_{2}, 7$ $\mathrm{MgCl}_{2}, 25$ glucose, and 75 sucrose saturated with $95 \% \mathrm{O}_{2}$ and $5 \% \mathrm{CO}_{2}$. After cutting, we recovered the slices for $30-45 \mathrm{~min}$ at $35^{\circ} \mathrm{C}$ and for $1 \mathrm{~h}$ at room temperature in the recording solution. The physiological extracellular solution used for recordings containing $(\mathrm{mM}): 125 \mathrm{NaCl}, 25 \mathrm{NaHCO}_{3}, 25$ glucose, $2.5 \mathrm{KCl}, 1.25 \mathrm{NaH}_{2} \mathrm{PO}_{4}, 2 \mathrm{CaCl}_{2}$, and $1 \mathrm{MgCl}_{2}$ (bubbled with $95 \% \mathrm{O}_{2}$ and $5 \% \mathrm{CO}_{2}$ ). For all experiments, we used an intracellular solution containing (in $\mathrm{mM}$ ): $126 \mathrm{~K}$ gluconate, $4 \mathrm{NaCl}, 1 \mathrm{MgSO}_{4}, 0.02 \mathrm{CaCl}_{2}, 0.1$ BAPTA, 15 Glucose, 5 HEPES, 3 ATP, and 0.1 GTP. The $\mathrm{pH}$ was adjusted to 7.3 with $\mathrm{KOH}$, and the osmolarity was adjusted to 290 mosmol $1^{-0}$ with sucrose.

Patch-clamp recordings. Neuronal firing activity and excitatory postsynaptic currents (EPSCs) were studied by whole-cell current-clamp and voltage-clamp recordings. All experiments were performed on morphologically identified layer $\mathrm{V}$ pyramidal neurons. Both approaches were performed using a Multiclamp 700B amplifier (Axon Instruments, Molecular Devices, Sunnyvale CA, USA) and an upright BX51WI microscope (Olympus, Japan) equipped with Nomarski optics. For the extracellular stimulation of layers II-III, we used a bipolar tungsten electrode (intensity: $50-150 \mu \mathrm{A}$, duration: $30 \mu \mathrm{s})$ connected to an isolated pulse stimulator (A-M Systems, Carlsborg WA, USA). Patch electrodes, fabricated from thick borosilicate glasses, were pulled and fire-polished to a final resistance of 5-7 M 2 . Recordings with either leak currents $>100 \mathrm{pA}$ or a series resistance $>15 \mathrm{M} \Omega$ were discarded. Experiments were performed at $22-24^{\circ} \mathrm{C}$. For the isolation of EPSCs in the voltage-clamp configuration, Bicuculline $(30 \mu \mathrm{M})$ and CGP58845 $(5 \mu \mathrm{M})$ were added to the extracellular solution. For the recording of miniature NMDA PSCs, TTX $(300 \mathrm{nM})$, CNQX $(10 \mu \mathrm{M})$, Bicuculline $(30 \mu \mathrm{M})$, and CGP58845 $(5 \mu \mathrm{M})$ were added to the extracellular solution. Moreover, the extracellular $\mathrm{Ca}^{2+} / \mathrm{Mg}^{2+}$ ratio was changed ( $2.5 \mathrm{mM} \mathrm{CaCl}_{2}$ and $0.5 \mathrm{mM} \mathrm{MgCl}_{2}$ ) to avoid the $\mathrm{Mg}^{2+}$ block of NMDA receptors.

\section{Peak Interval-Fixed Interval Behavioral Assay}

Apparatus and procedure. In this study, we used a device called COWE (Cognition and Welfare), which was developed by TSE Systems (Germany) on the basis of the PhenoMaster System (Urbach et al, 2010) and consists of an operant wall located in the home cage. The operant wall has three hopper/ holes with infrared beams that detect the nose-poke (NP) activity, but in this case, we blocked one lateral hopper and we used two hoppers, one next to the other. The right hole was the control hopper, while the left hole was connected to a food dispenser/feeder that released $20 \mathrm{mg}$ of dustless precision pellets (BioServ, USA). Each hole was equipped with a small LED with 4 mcd (millicandela) of luminous intensity that was remotely controlled via computer, as it was for the infrared beams and feeder. The TSE Systems-OBS software was used to design trial-by-trial protocols for group cages. The animals were kept in light-controlled, sound 
proof, and ventilated cubicles. The cubicle light (100-110 lux) was set to switch on at 7 a.m. and to switch off at 7 p.m.

Experimental design. Before starting the experiments, the animals were singly housed and adapted to the precision pellets with food restriction for 2 days. The experiment consisted of a Pre-Training phase and a Training phase. In the Pre-Training phase, the mice self-initiated the trial by poking in the right hopper, determining the illumination of the two holes. The animals were required to NP in the left hopper after the light signal went off $(L=2 s)$ to receive a pellet reward. During this phase, the animals learned the association between the left hopper and the feeder. For each trial, there was a $30 \mathrm{~s}$ time limit within which mice could receive a reward. At the end of each trial, the Inter Trial Interval began, which was the sum of a fixed interval of $30 \mathrm{~s}$ and a random interval coming from a geometric distribution with a mean of $60 \mathrm{~s}$. This phase lasted 3 days. The Training phase consisted of a variant of the Peak Interval timing procedure with $80 \%$ Fixed-Interval (FI) trials and $20 \%$ unreinforced probe trials. During the FI trials, the mice, as in the Pre-Training phase, self-initiated the trial by poking in the right hopper $(\mathrm{R})$, determining the illumination of the two holes. After $2 \mathrm{~s}(\mathrm{~L}=2 \mathrm{~s})$ from the beginning of the trials, the lights were switched off, and only after $10 \mathrm{~s}(\mathrm{~T}=10 \mathrm{~s})$, a pellet reward was delivered in response to a NP in the left hopper (L). Any NP before $10 \mathrm{~s}$ had no consequence, and any NP after the time limit $(30 \mathrm{~s})$ was not reinforced. The Inter Trial Interval began after the release of the pellet or at the end of the time limit. Twenty percent of the probe trials $\left(\mathrm{L}_{\mathrm{p}}\right)$ were randomly intermixed with the normal trials and were identical to the FI trials, but without payoff. This phase lasted 5 days.

For Data Analysis, see SI Materials and Methods.

\section{Schedule of a Reinforcement Fixed Interval $30 \mathrm{~s}$ in Mice}

Apparatus. Standard operant conditioning chambers (MED Associates, East Fairfield, VT) were enclosed within soundattenuating ventilated cubicles, connected to a computer through an interface and controlled by MED-PC software. Chambers were equipped with a white house light centered $10 \mathrm{~cm}$ above the NP manipulanda (positioned $2 \mathrm{~cm}$ above the floor) and a food dispenser, which delivered 14-mg food pellets (5TUL-1811215 IPS Product Supplies, London, ENG).

Pre-training. Male 8-week-old C57BL/6J mice and additional group of WT and TAAR1-KO mice were deprived of food and trained to NP for food during two consecutive daily sessions (habituation and magazine training).

Training. Mice were trained to NP for food pellet delivery according to a fixed-ratio 1 schedule of reinforcement. Only one NP in each box was active. A response in this NP was recorded (correct response) and rewarded by the delivery of one food pellet. A response in a different NP was scored as an incorrect response, but did not have programmed consequences. The left-right locations of the active and inactive NPs were counterbalanced in the mice. The position of active NPs for each mouse was constant for the duration of the experiment. The criterion for NP response acquisition included 50 reinforcements earned during the 30 -min session.
FI schedule. After mice acquired the NP response (4 days), the FI $3 \mathrm{~s}$ schedule was started. The FI schedule of reinforcement involved delivering reinforcement for the first response after a specific period of time had elapsed. In the FI-3 s, a correct response before $3 \mathrm{~s}$ after the previous delivery of reinforcement expired was scored as a premature correct response but did not have programmed consequences. The FI schedule was gradually increased: FI-5 s, FI-8 s, FI-12 s, FI-18 s, FI-24 s, and FI-30 s. The duration of the session was limited to $60 \mathrm{~min}$. Subjects received one session per day. Drug tests were started when the mice stabilized their behavior, showing less than 20\% variability during the last 2 days before the test.

Drug tests. RO5166017 (0.1-1.0 mg/kg) and RO5203648 $(0.03-0.3 \mathrm{mg} / \mathrm{kg})$ were dissolved in saline with minimal amounts of Tween 80 and administered i.p. in a $10 \mathrm{ml} / \mathrm{kg}$ injection volume. All drugs were given $30 \mathrm{~min}$ prior to the test session. In the FI test, different doses were tested in the order derived from a Latin square design.

Data analysis. The primary dependent variables were the number of premature correct NP responses and the postreinforcement pause (time between last reinforced correct NP response and next correct NP response). Additional analysis was conducted on the number of NPs in the food tray.

\section{RESULTS}

\section{Expression of TAAR1 in Cortical Neurons}

Ventral tegmental area dopaminergic neurons project to different subregions of the PFC, making synapses with glutamate and GABA neurons located in different layers (Tritsch and Sabatini, 2012). TAAR1 is expressed in mouse ventral tegmental area dopaminergic neurons, and according to some preliminary reports, it might be found in the frontal cortex (Lindemann et al, 2008; Di Cara et al, 2011). To understand the role that TAAR 1 could have in this region, we first evaluated its expression in the PFC of adult mice. Using RT-PCR, we determined that TAAR1 mRNA is indeed found in the PFC, with no signal detected in TAAR1-KO mice (Supplementary Figure S1). We also measured the TAAR1 transcript in adolescent mice (1-month old), to evaluate the whether TAAR1 is differentially expressed during the development. As shown in Supplementary Figure S1, both adolescent and adult animals express similar levels of TAAR1 transcript. Furthermore, to investigate the precise localization of TAAR1 in this area, we have additionally used a recently developed transgenic rat model, TAAR1 KO/dsRed knock in rats, where the fluorescent marker dsRed was expressed under TAAR1 promoter allowing mapping of TAAR1 expression in neuronal populations with an accuracy that is not achievable currently in mice (Harmeier and Hoener; manuscript in preparation). By using this novel tool that allows both the investigation of the consequences of the lack of TAAR 1 in rats and the reliable mapping of TAAR1 expression pattern, we found a distinct fluorescent signal in layer $\mathrm{V}$ cortical neurons in general and in PFC specifically (Figure 1a, right panel, bottom). No signal was detected in other layers of cortex of transgenic rats and any region of WT animals (Figure 1a, right panel, top). 
a


b

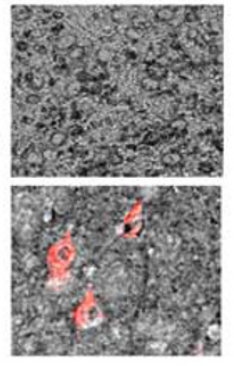

$\circ W T$
$\circ K O$

d
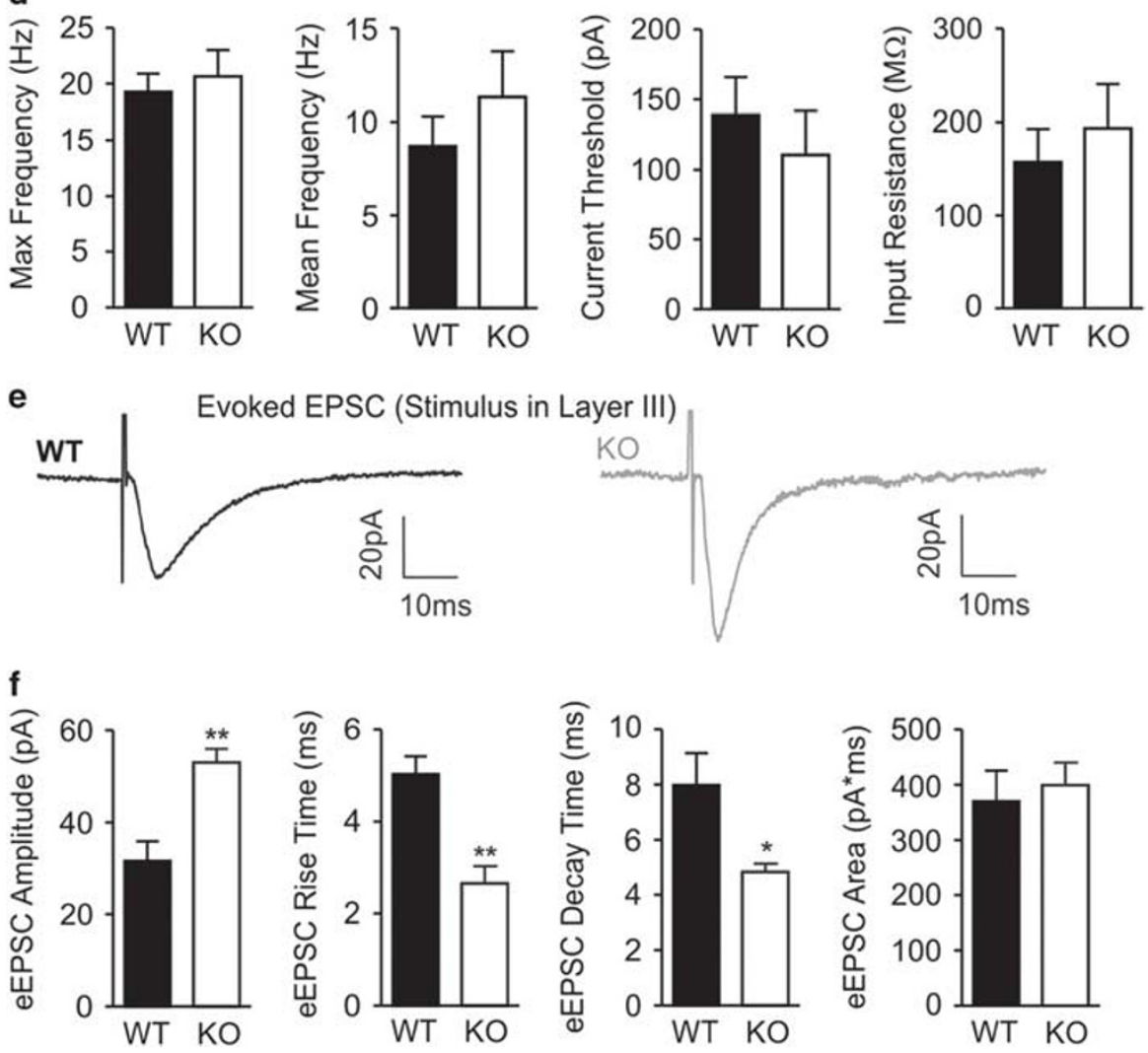

Figure I Deletion of TAARI increases the amplitude and kinetics of evoked excitatory postsynaptic currents in layer $V$ pyramidal neurons of the mouse frontal cortex. (a, right panel) The expression of the red fluorescent protein dsRed under the rat TAARI promoter in the layer $V$ of PFC of transgenic TAARI $\mathrm{KO}$ dsRed rats. Representative images were taken using the overlay of the bright-field and dsRed channel from the layer $\mathrm{V}$ of PFC of transgenic TAARI KO dsRed rats (a, right panel, bottom) and WT rats (a, right panel, top). (a, left panel) Nissl-stained coronal section of mouse brain slice containing the medial prefrontal cortex (mPFC; box) in which patch clamp experiments were performed (a). Current-clamp protocol and representative traces of action potentials induced by the injection of $200 \mathrm{pA}$ (b). Action potential frequency vs injected current relationship (F/l curve) and resting membrane potential (c), maximal firing rate, mean firing frequency, current threshold, and input resistance (d) were measured in WT and TAARI-KO mice. (e) Representative traces of evoked excitatory postsynaptic currents from layer $\mathrm{V}$ pyramidal neurons recorded upon stimulation of layer III neuron fibers in both WT and TAARI-KO mice. (f) Amplitude, rise time, and decay time of evoked excitatory postsynaptic currents were altered in TAARI-KO compared with WT neurons. Data represent means \pm SEM. $N=8-12$ cells per group and $N=3-4$ mice per group. $* p<0.05$; ${ }^{*} * p<0.0$ I. Student's unpaired two-tailed t-test. 

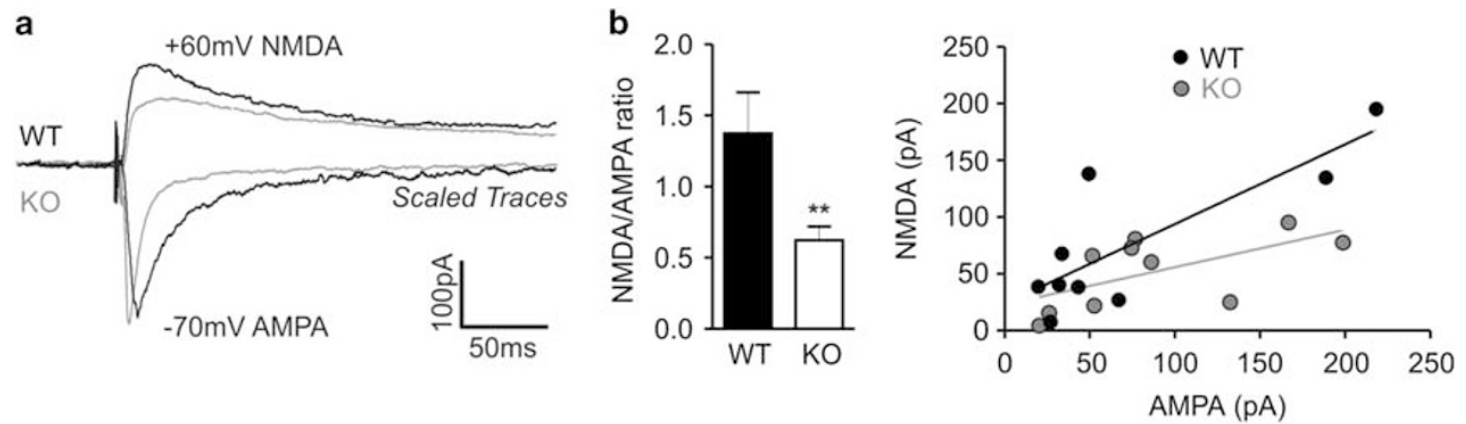

C
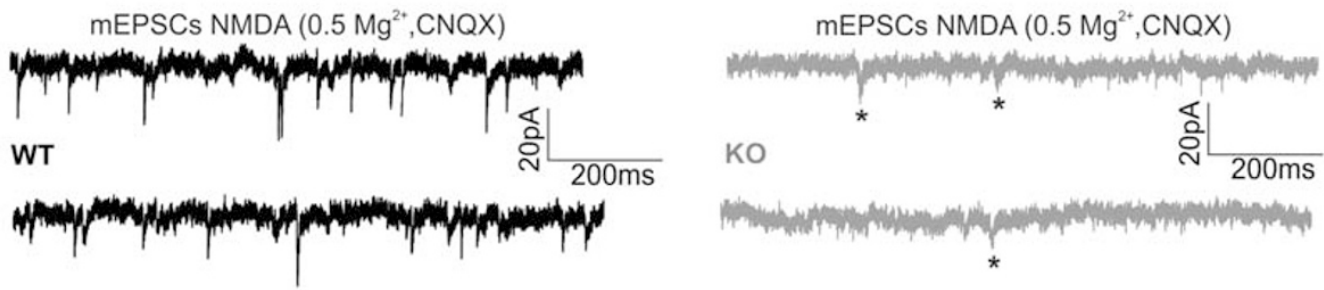

d

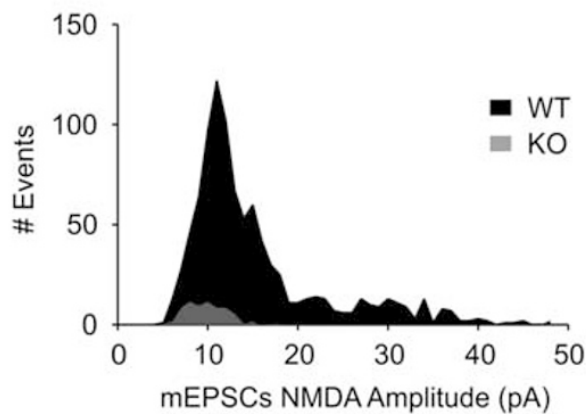

e

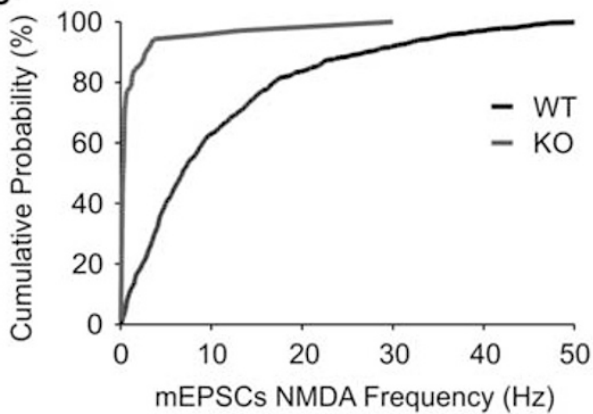

Figure 2 TAARI regulates NMDA receptor number/efficiency in layer $V \mathrm{mPFC}$ neurons in mice. Representative traces of evoked AMPA ( $-70 \mathrm{mV}$ ) or NMDA (+60 mV) excitatory postsynaptic currents from layer $V$ pyramidal neurons recorded upon stimulation of layer III fibers (a). The NMDA/AMPA ratio decreases in TAARI-KO neurons with respect to WT neurons (b). Representative traces of miniature NMDA excitatory postsynaptic currents from both WT and TAARI-KO layer $V$ mPFC neurons (c). TAARI-KO neurons show a decrease in NMDA miniature postsynaptic current amplitude and frequency ( $\mathrm{d}$ and e). Data represent means \pm SEM. $N=6-10$ per group $N=3-4$ mice per group. ${ }^{*} * 0.0$ I Student's unpaired two-tailed $t$-test.

Taken together, these observations indicate a significant expression of TAAR1 in rodent PFC neurons.

\section{Deficient Glutamate NMDA Receptors Function in the mPFC Layer V Pyramidal Neurons of TAAR1-KO Mice}

To further evaluate the role of TAAR1 in this area, we applied patch-clamp electrophysiological approach to cortical slices. First, we analyzed the impact of TAAR1 deletion on the intrinsic neuronal excitability of layer $\mathrm{V}$ pyramidal neurons in the mPFC (Figure 1a, left panel). For this purpose, we used the patch-clamp method in the current-clamp configuration, and we measured the output of the WT and TAAR1-KO mPFC neurons in response to current injection steps (Figure 1c). The injection of current did not modify the action potential firing frequency in TAAR1-KO neurons with respect to WT neurons (Figure $1 \mathrm{~b}$ and $\mathrm{c}$ ), and it did not alter other parameters of neuron excitability (Figure 1d). However, the membrane resting potential in TAAR1-KO neurons was more depolarized compared with WT neurons (Figure 1c), suggesting a potential effect at the level of synaptic contacts in the dendritic tree of these neurons. Synaptic inputs on pyramidal neurons are important for the signal integration that elicits an action potential; thus, the next step was to analyze, in detail, the excitatory synaptic strength of TAAR1-KO mPFC neurons. After extracellular stimulation of layers II-III of the mPFC with a bipolar tungsten electrode, we found that the amplitude of EPSCs is increased in TAAR1-KO neurons (Figure 1e and $\mathrm{f}$ ). Moreover, the kinetics of EPSCs was altered in the TAAR1-KO neurons (Figure 1f), suggesting possible changes in the composition of postsynaptic glutamatergic receptors.

To investigate which postsynaptic receptor subtypes could be involved in the alteration of EPSC kinetics in TAAR1-KO mPFC neurons, we analyzed the NMDA/AMPA ratio in the mPFC layer $\mathrm{V}$ pyramidal neurons upon stimulation of the layer III neuron fibers. We found that this ratio was altered in TAAR1-KO neurons (Figure 2a and b), most likely owing to a reduction of the NMDA currents. To test this hypothesis, we performed miniature NMDA excitatory postsynaptic currents and found a strong decrease in both the amplitude and the frequency of these currents in the TAAR1-KO neurons (Figure 2c-e). These data indicate the NMDA receptor-dependent synaptic deficits in $\mathrm{MPFC}$ layer $\mathrm{V}$ pyramidal neurons of mice lacking TAAR1. 

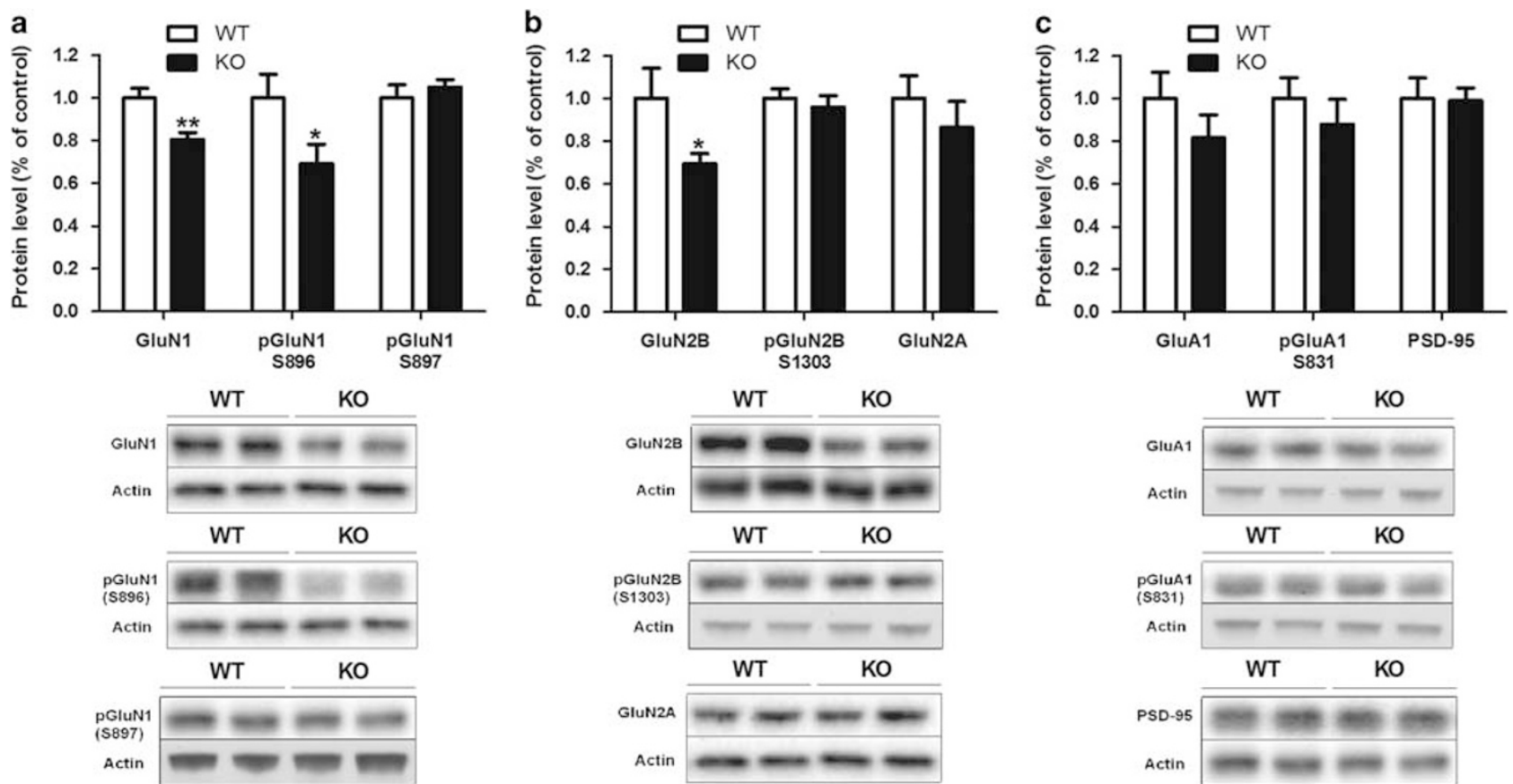

Figure 3 NMDA receptor composition is altered in the prefrontal cortex of mice lacking TAARI. Western blot densitometric analysis of the prefrontal cortex extracts from WT and TAARI-KO mice. Synaptosomal fractions were separated from the whole lysate and then analyzed by gel electrophoresis. Antibodies against GluNI (a), p-S896- GluN I (a), p-S897- GluN I (a), GluN 2B (b), p-SI 303- GluN 2B (b), GluN 2A (b), GluAI (c), p-S83I-GluAI (c), and PSD-95 (c) were used. Actin was used as the loading control for the densitometric analysis. Results were normalized to their respective WT controls. Data represent means \pm SEM. $N=10$ - 15 per group. $* p<0.05$; $* * * 0.01$. Student's unpaired two-tailed $t$-test.

\section{Glutamate NMDA Receptor Composition is Altered in the PFC of TAAR1-KO Mice}

NMDA receptors are hetero-tetramers formed from the assemblies of GluN1 subunits with GluN2A-D and GluN3 A/B (Collingridge et al, 2013). Thus, to study the molecular determinants responsible for the alterations seen in electrophysiological experiments, we evaluated the expression of several proteins that are critical for glutamate synapses in the synaptosomal fraction of the PFC of adult WT animals and their KO littermates by using the western blot approach. In these experiments, we found a decrease in the expression of the GluN1 subunit (Figure 3a), as well as a decrease in the phosphorylation of GluN1 at serine 896 (Figure 3a), in the PFC of TAAR1-KO mice. No change in the serine 897 residue of the same subunit was observed (Figure 3a). Furthermore, a decrease in the expression of GluN2B was also documented (Figure $3 \mathrm{~b}$ ), with no change observed in phospho-GluN2B (serine 1303) (Figure 3b) or GluN2A levels (Figure 3b). In contrast to GluN1 and GluN2B NMDA receptor subunits, AMPA receptor subunit GluA1 levels and phosphorylation status (Serine 831) were not changed in mutants (Figure 3c). Similarly, the levels of PSD-95, a postsynaptic protein critically involved in the proper organization of postsynaptic structure and integrity (Gardoni and Di Luca, 2006), were not altered by TAAR1 deficiency (Figure 3c). These data demonstrate significant alterations in the composition of cortical NMDA receptors further supporting NMDA receptor dysfunction observed in the PFC of TAAR1-KO mice in electrophysiological experiments. In addition, to test whether TAAR1 activation could modulate NMDA activity acutely, we used another experimental approach by using cultured cortical neurons. Cultured neurons were treated with RO5166017 at $1 \mu \mathrm{M}$ or vehicle, and the expression of GluN1 and its phosphorylation site S896 was assessed by western blot. As shown in Supplementary Figure S2, RO5166017 produce a modest increase in GluN1 expression but a significant increase in the phosphorylation of S896, suggesting that TAAR1 can influence NMDA activity acutely.

\section{Lack of TAAR1 Leads to Perseverative and Impulsive Behaviors}

To unveil the behavioral consequences of TAAR1 deficiency leading to altered cortical NMDA receptor function, we first evaluated the cognitive functions of TAAR1-KO mice in a Fixed Interval-Peak Interval test (Matell and Meck, 2000). The automated protocols allowed the testing of mice in home cages for $24 \mathrm{~h}$ per day for several days to collect a massive amount of data with less variability by monitoring mouse behavior according to their physiological sleep-wake cycle. In particular, this test allows reliable assessment of various aspects of cognitive functions of mice in home cage environment. All mice received 3 days of magazine training to familiarize the mice with reward delivery. Overall, the animals managed to gain more than 160 pellets per day before switching to the Training phase. During the training phase (Figure $4 \mathrm{a}$ and $\mathrm{b}$ ), the NP activity was monitored; such activity has a circadian oscillation along the days according to the light/dark cycle (Figure 4c). The amount of activity was computed with $1 \mathrm{~h}$ bin for each subject, and the 
a
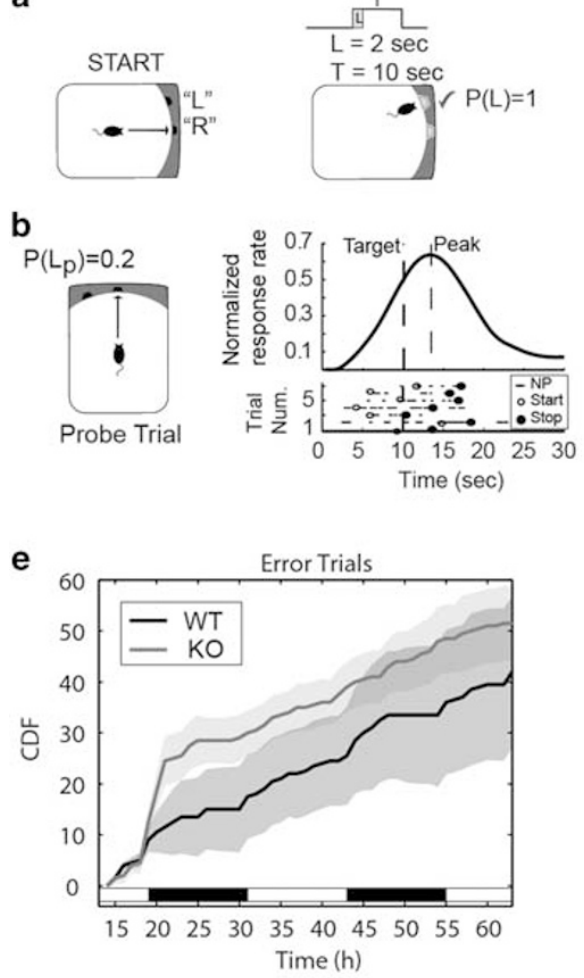

h

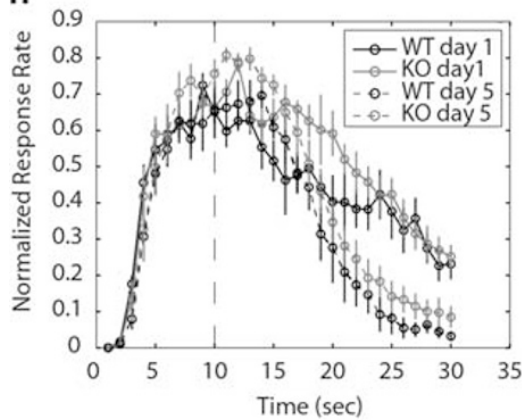

C

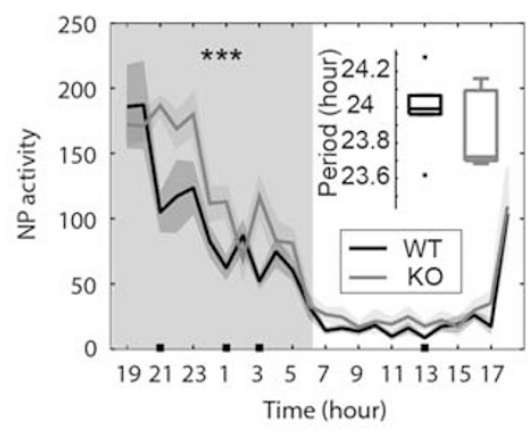

f

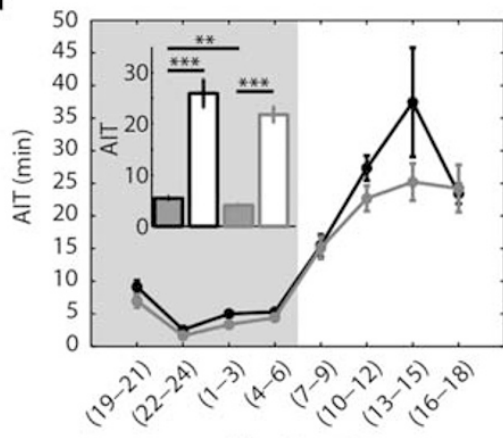

Time (hour)

i

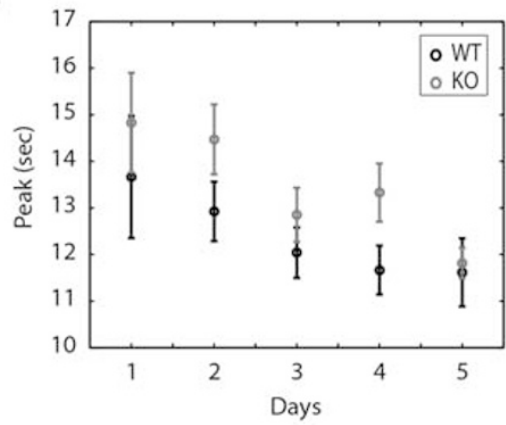

d

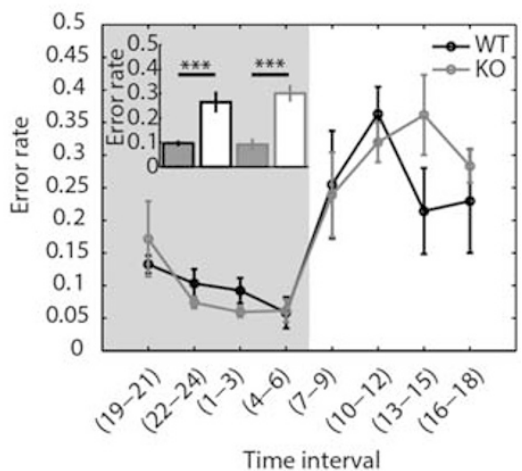

g

g Start\&Stop Dark phase

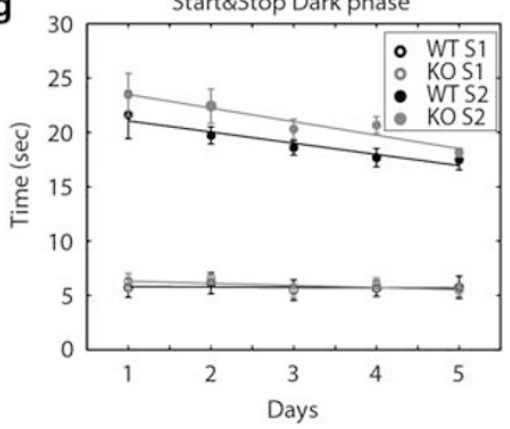

j

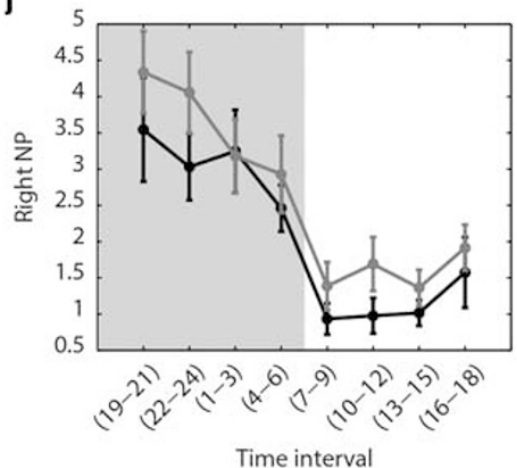

Figure 4 TAARI-KO mice display perseverative and impulsive behaviors in home cage. Graphical representation of the task. Fix-Interval (FI) task (a) presents two hoppers (right ' $R$ ' and left ' $L$ ') in which a light signal of two seconds $(L=2)$ declares the start of the trial. The target time ( $T$ ) is fixed to I 0 s. The probability of trial to occur in ' $\mathrm{L}$ ' location is $100 \%(\mathrm{P}(\mathrm{L})=\mathrm{I})$. Peak-Interval $(\mathrm{PI})$ task $(\mathrm{b})$ introduces probe trials $\left(\mathrm{L}_{p}\right)$ that are randomly intermixed to normal trials with $20 \%$ of probability $\left(P\left(L_{P}\right)=0.2\right)$ to occur (left panel). Example of normalized response curve during probe trials (right panel). The circadian profile of the nose-poke (NP) activity (c) exhibits significant genotype (two-way ANOVA: $F(I, \mid 43)=|3.7|$, **** $p<0.00$ I) difference during the dark phase (I9-7 h). (c). Boxplots above the curves show no difference in the circadian periodicity for WT and TAARI-KO mice (c). The circadian trend of the error rate (d) was significantly different within genotype and between light and dark phases (two-way ANOVA for WT: $F(I, I 43)=40.67$, p < 0.00 I; for KO: $F(I, I 43)=64.96$, $p<0.00 \mathrm{I})$, but not between genotypes $(F(I, 287)=0.47, p=0.49)$ as summarized in the histogram above the curve. Cumulative distribution function $(C D F)$ of the error rate along the time (e). Significant difference of the total errors between genotypes during the first $3 \mathrm{~h}$ of the first dark phase (I $9-2 \mathrm{I})$, $p=0.04$. No difference between genotypes in the subsequent intervals $(p \geqslant 0.05)$. Analysis of the actual inter-trials (AITs) ( $f$ ) revealed a difference between genotypes along $24 \mathrm{~h}$ (two-way ANOVA: $\mathrm{F}(\mathrm{I}, 287)=4.5, p=0.03$ ) that was accentuated during the dark phase $(\mathrm{F}(\mathrm{I}, \mathrm{I} / 43)=8.8 \mathrm{I}, p=0.003)$. Bonferroni post hoc analysis does not reveal significant difference between each time point. Analysis of START (SI) and STOP (S2) responses (g). During the dark phase, the STOP times changed significantly across days (two-way ANOVA: $F(4,56)=3.64, p=0.01$ ). The genotype effect was also significant $(F(I, 59)=5.35, p=0.02)$. There was no difference in the START time. Normalized response rate during probe trials in the first and last days $(h)$. Peak times were significantly different between genotypes $(F(I, 59)=4.19, p=0.04)$ and showed significant changes over time $(F(4,56)=3.05, p=0.02)$ (i). The right NPs during the AlTs, along the $24 \mathrm{~h}$, revealed a difference between genotypes $(F(I, 96)=4.44, p=0.03)(j)$. All the data are shown as mean \pm SEM per group $(N=6$ per group for both WT and TAARI-KO mice). $*$ p $<0.05$; $* * * 0.005$; $* * * * 0<0.00$ I.

periodicity, computed with a periodic fit (see Materials and Methods), was perfectly entrained with the light/dark cycle (Figure 4c, boxplots above the graph). The curves showed a significant increase in the NP activity for TAAR1-KO mice during the dark phase, suggesting hyperactivity during the night, but not during the light phase (Figure 4c). The circadian trend of the error rate was significantly different within the genotype between the light and dark phases but 
not between the genotypes (Figure 4d). Interestingly, by analyzing error trials during the first day of test, it was evident that TAAR1-KO mice were slower in the learning of the test (Figure 4e). During the first hours of the test, when mice were learning how to get the reward, TAAR1-KO mice performed worse than WT mice, shown as the difference in the slope of the cumulative curve of error trials (Figure 4e). Later, when the test was learned, the slopes between the two groups were similar (Figure 4e). To further assess the changes of the performance over the light/dark cycle, we analyzed the Actual Inter-Trials (AIT) durations between two consecutive trials. These intervals occur because of the delay of the mouse to initiate a trial and have been used as a measurement of impulsivity (Matell and Portugal, 2007). A significant difference was found between genotypes along the $24 \mathrm{~h}$ and was accentuated during the dark phase (Figure 4f). Furthermore, the distribution of the response rate during the probe trials was analyzed, as well as the acquisition of the START (S1) and STOP (S2) responses. During the dark phase, the S2 times changed significantly across days (Figure 4g), whereas the $\mathrm{S} 1$ times remained constant and equal between genotypes, highlighting a perseverative pattern of behavior in TAAR1-KO mice. The improvement in the acquisition of the STOP responses was evident by the increase in the slope of the right tail of the normalized response rate (Figure $4 \mathrm{~h}$ ) from the first to the fifth day of the experiment. Although there was an improvement in STOP responses between the first and last days in both TAAR1-KO and WT mice, a significant difference between the two genotypes remained throughout the experiment (Figure 4h). Moreover, the Peak times, which reflect the accuracy in the timing perception, were significantly different between the genotypes (Figure 4i) and showed significant changes over time. These changes in the temporal precision and accuracy (Figure $4 \mathrm{~g}-\mathrm{i}$ ) are considered the hallmarks of the clock-speed adjustment (Matell et al, 2006). The right hopper was assigned to the activation of the trial after a refractory period, the AIT. To further analyze impulsive behavior in these mice, we analyzed the average number of NPs in the right hopper during the AITs, after the release of the reward and along the $24 \mathrm{~h}$ because this type of observation has also been considered to be related to impulsivity (Dalley et al, 2004). According to the decrease in the AITs, we observed a significant increase in the number of NPs in KO mice compared with control animals (Figure 4j). Thus, we observed several indications of cognitive abnormalities in mutant mice further supporting altered cortical function in mice lacking TAAR1.

\section{TAAR1 Agonists Reduce Impulsivity in Mice}

To test whether we could modulate behaviors generally related to mPFC function by pharmacological activation of TAAR1, we examined the effects of two TAAR1-selective ligands (Revel et al, 2013), a full agonist and a partial agonist, RO5166017 and RO5203648, respectively, (Revel et al, 2011; Revel et al, 2012) in a classic Skinner's schedule of reinforcement, FI-30 s (Sagvolden et al, 1983) in C57Bl/6J mice. This behavioral paradigm provides a measure of impulsivity that is believed to be at least in part controlled by cortical function (Bari and Robbins, 2013). After training with increasing periods of intervals (from 3 to $30 \mathrm{~s}$ ), the test started. C57B1/6J mice were injected with saline or TAAR1 agonists before the session, and two parameters were scored as a measure of impulsivity, including premature correct responses and the post-reinforcement pause (Berger and Sagvolden, 1998). Pretreatment with RO5166017 and RO5203648 decreased the number of correct premature responses (Figure 5a and c). By analyzing the post-reinforcement pause, we observed a trend towards an increase in this value, although it did not reach statistical significance (Figure $5 \mathrm{~b}$ and $\mathrm{d}$ ). To further validate the target specificity of these effects, we performed an additional experiment with the highest dose of RO5203648 in WT and TAAR1-KO animals. In agreement with a previous experiment (Figure $5 \mathrm{c}$ and $\mathrm{d}$ ), this TAAR1 ligand significantly decreased the premature correct responses (Figure 5e) and increased postreinforcement pause $(5 F)$. In TAAR1-KO mice, the number of premature correct responses did not differ from WT mice, however, RO5203648 failed to exert any effect on both parameters in mutant mice (Figure 5e and f). Notably, in agreement with previous studies (Revel et al, 2011; Revel et al, 2012), these compounds did not exert sedative effect as indicated by the analysis of the total NPs made in the central hole and the total reinforcements obtained during the test session (Supplementary Figure S3). Both RO5166017 (Supplementary Figure S3A and B) and RO5203648 (Supplementary Figure S3C and D) did not change these parameters significantly. All together, these data suggest that pharmacological activation of TAAR1 in vivo modulates impulsivity in mice.

\section{DISCUSSION}

In this study, we demonstrated that the lack of TAAR1 results in reduction of glutamate NMDA receptor activity indicating that TAAR1 can modulate PFC-related processes and behaviors. First, we observed that TAAR1 is expressed in the PFC, primarily in the pyramidal neurons of layer $\mathrm{V}$, supporting and extending the previous evidence on TAAR1 mRNA expression in the frontal lobe (Di Cara et al, 2011). Furthermore, by using patch clamp recording in the pyramidal neurons of layer $\mathrm{V}$, we found an altered response upon stimulation of the afferent fibers. From the analysis of the eEPSC, we noted that there was an increase of the current that potentially may suggest an increase in AMPA receptor activity. At the same time, a decrease of the current kinetic may suggest a decrease in NMDA receptor activity. Moreover, we found a decreased NMDA/AMPA receptors ratio, which may indicate glutamate NMDA receptor hypofunction. The analysis of the mEPSCs mediated by NMDA revealed a strong reduction in the frequency and amplitude of the events, indicating indeed that postsynaptic NMDA receptors in these layers were less active. These data are intriguing because the dysfunction of layer $\mathrm{V}$ pyramidal neurons in the PFC has been correlated with cognitive deficits (Seamans et al, 2001). It is known that NMDA receptor function can negatively affect AMPA current and spine formation/morphology at pre- and postsynaptic side (Ultanir et al, 2007). In particular, the GluN2B subunit was shown to inhibit AMPA receptor surface expression (Kim et al, 2005). Thus, the increase of AMPA current observed in our experiments may be potentially explained by the 

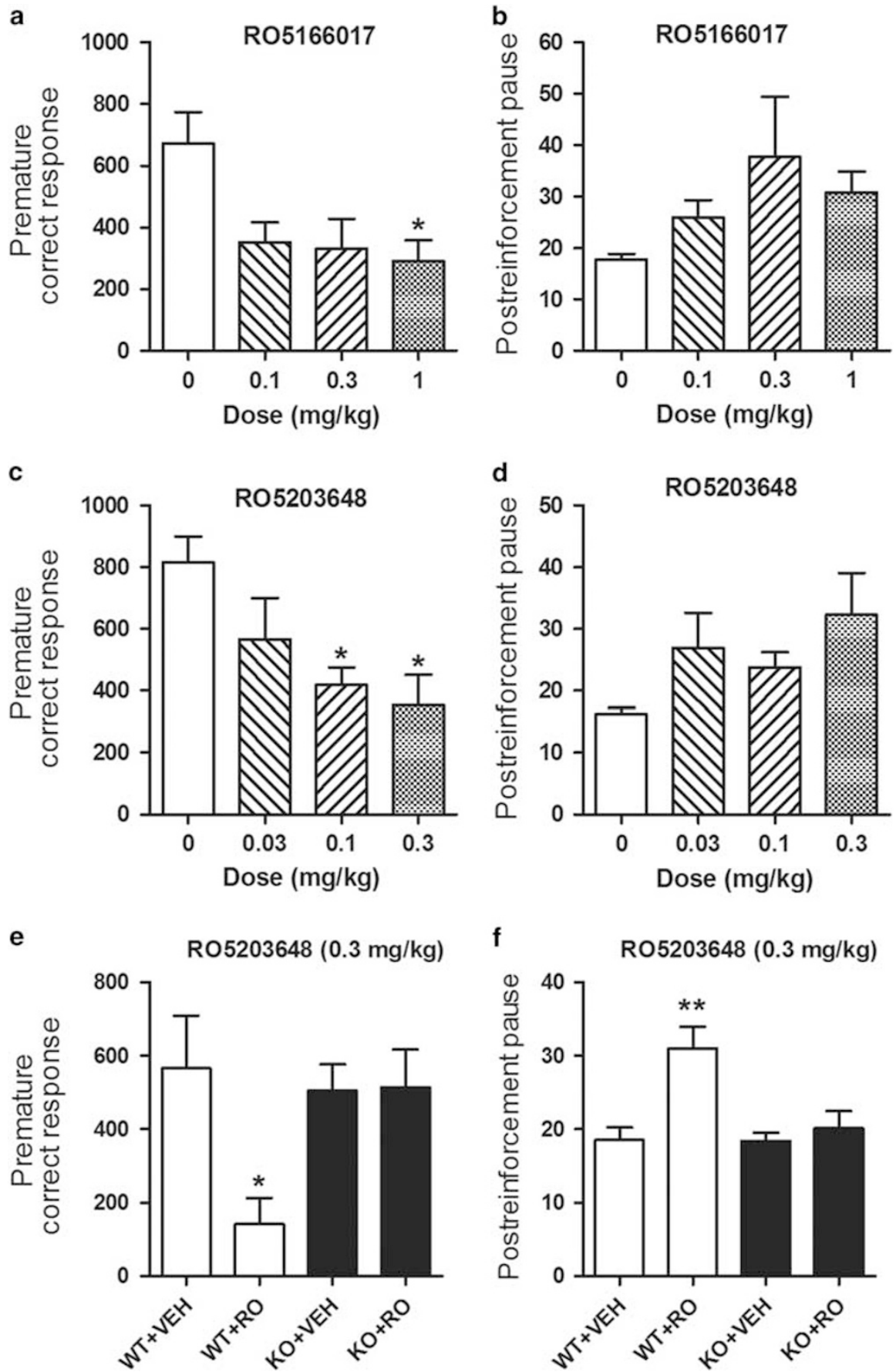

Figure 5 Systemic administration of selective TAARI agonists reduces impulsivity in a Fl 30 s test in normal mice RO5 I 660 I7 and RO5203648 decreased the number of correct premature responses in C57BI/6) mice. ( $(a$ and $c) ; F(3,28)=4.078 p<0.05$ and $F(3,26)=8.029 p<0.05$, respectively). Post hoc analyses (Bonferroni's test) revealed that this TAARI agonist effect was statistically significant at the dose of $1.0 \mathrm{mg} / \mathrm{kg}$ for RO5I660I7 and at the doses of 0.1 and $0.3 \mathrm{mg} / \mathrm{kg}$ for RO5203648. Activation of TAARI receptors did not affect significantly post-reinforcement pause $(F(3,28)=1.967 p=0.14 ; F(3,26)=2.506$ $p=0.08, \mathrm{RO} 5 \mathrm{I} 66017$ and RO5203648, respectively) (b and d). All the data are shown as mean $\pm \mathrm{SEM} . \mathrm{N}=12$ per group. RO5203648 at dose $0.3 \mathrm{mg} / \mathrm{kg}$ was able to reduce the number of correct premature responses in WT $(p<0.05)$ but not in TAARI-KO animals (e). Similarly, RO5203648 increased postreinforcement pause in WT $(p<0.0 \mathrm{I})$ but not in TAARI-KO mice ( $)$. All the data are shown as mean \pm SEM. $N=10$ (WT) and 8 (KO) per group. * $p<0.05$; $* * * 0.0$ I. One-way ANOVA with Bonferroni post hoc test.

reduction of the GluN2B subunit that finely regulates AMPA receptor availability at the postsynaptic side membrane without significant changes in the total amount of AMPA receptor protein. Furthermore, the decrease in NMDA receptor function may alter the presynaptic side increasing the release of glutamate from synaptic vesicles and consequently the AMPA current.
In an attempt to identify the molecular alterations that may contribute, at least partially, to such abnormalities, we investigated the levels and the phosphorylation state of key proteins of the glutamate synapse in the PFC of TAAR1-KO mice. We found that the expression of GluN1 and GluN2B subunits of NMDA receptors was decreased, with a concomitant decrease in the phosphorylation site on the 
GluN1 subunit (S896). This is of note given that GluN1 downregulation has been linked to a schizophrenia-like phenotype and is speculated to be intimately involved in related cognitive deficits (Mohn et al, 1999). It should be noted that although we have not noted significant alterations in AMPA receptor levels and phosphorylation, we cannot exclude an involvement of AMPA receptors in TAAR1mediated functions, that might be related to potential alterations in the availability of AMPA receptors in cellular microdomains or in synaptic $v s$ extrasynaptic sites.

These results were further corroborated by testing cognitive function of TAAR1-KO mice in home cage environment in a Fixed Interval-Peak Interval test (Matell and Meck, 2000). Particularly, we attempted to unveil whether and which type of cognitive deficits may arise from TAAR1 ablation. We found, in fact, that the lack of TAAR1 per se did not cause a global cognitive impairment but these mice were making more errors during the first period of learning, indicating a small initial deficit in learning. However, even more interestingly, these mice displayed an incorrect time of perception, as indicated by the analysis of the peaks during probe trials (Coull et al, 2011). Timing is a cognitive property well conserved between species, and the PFC plays an essential role in mediating the correct timing perception (Meck et al, 2008). By analyzing the STOP responses during the probes trials, it emerged that TAAR1-KO mice have delayed average STOP responses. It is also clear from Figure $4 j$ that the shape of the NP distribution between the two genotypes was different, with a wider difference on the first day that was still evident on the last day. This perseverative behavior, defined as a lack of suppression of previously engaged and ineffective behavior, is a sign of impairment in cognitive flexibility and could be modeled by pharmacological treatments that alter PFC functions as well as by frontal cortex lesions (Floresco et al, 2009). Notably, this particular deficit is also a phenotypic trait of several psychiatric diseases, such as schizophrenia, obsessive-compulsive disorder, and ADHD. From the analysis of the NPs during the AIT and the correlated duration of the AIT, we also observed a decrease in the AIT for TAAR1-KO mice and an increase in the NPs during this period, indicating an impulsive behavior in mice lacking TAAR1.

Furthermore, by using a classic Skinner's schedule of reinforcement, FI-30 s test, a validated experimental paradigm for impulsivity in mice (Sagvolden et al, 1983), we demonstrated that two selective TAAR1 agonists (Revel et al, 2013) reduced impulsive responding in normal mice thus indicating that stimulation of this receptor ameliorates impulsivity. Impulsivity has also been generally related to a PFC dysfunction and is found across several disorders, such as bipolar disorder, ADHD, and addiction (Bari and Robbins, 2013). Both full and partial TAAR1 agonists are known to be effective in reversing hyperactivity in pharmacologic or genetic models of hyperdopaminergia and NMDA deficiency in mice (Revel et al, 2012; Revel et al, 2013). Interestingly, TAAR1-KO mice are more prone to the addictive properties of methamphetamine, ethanol, and MDMA (Di Cara et al, 2011; Achat-Mendes et al, 2012; Lynch et al, 2013), while recent reports from different groups have demonstrated that TAAR1 full and partial agonists reduce cocaine-conditioned place preference, self-administration, and relapse (Revel et al, 2012; Pei et al, 2014; Thorn et al, 2014a; Thorn et al, 2014b). As impulsivity is considered to be a specific trait in the pathophysiology of drug addiction, it is possible that TAAR1 can reduce addictive behaviors in part through PFC-related mechanisms. Certainly, more studies are necessary to directly connect the observed PFC deficits and the impulsive/perseverative phenotype of mutant animals. Particularly, as TAAR1 is expressed in both the ventral tegmental area dopaminergic nuclei (Lindemann et al, 2008) and in the PFC (Figure 1a), the development of a conditional region-selective mutant mouse line would be critical to address this question.

With respect to the mechanism by which TAAR1 could modulate cortical glutamate NMDA receptor-related transmission and functions, several possibilities should be considered. It is known that TAAR1 exerts potent modulatory influence over mesolimbic dopaminergic system likely via D2 dopamine receptor-TAAR1 heterodimerization (Espinoza et al, 2011) and similar mechanisms could be involved in the regulation of cortical dopamine transmission as well. In this case, the observed alterations in cortical glutamate transmission could be secondary to alterations in cortical dopamine system. Alternatively, as TAAR1 is expressed in the layer V of PFC, where significant deficiency in NMDA receptor-mediated glutamate transmission was observed, it is possible that glutamate transmission deficits could be a direct consequence of the absence of cortical TAAR1. Intriguingly, a recent report indicated that TAAR1 can modulate the function of the glutamate transporter EAAT2 leading to alterations in glutamate clearance suggesting a direct connection between TAAR1 and glutamate transmission (Cisneros and Ghorpade, 2014). Finally, we cannot exclude an involvement of other monoaminergic systems and signaling pathways (ie, serotonin-mediated, (Lindemann et al, 2008)) that might be involved in TAAR1mediated functions and behaviors. Further studies are necessary to explore all these potential mechanisms.

\section{CONCLUSIONS}

In conclusion, we document here an abnormal PFC function in mice lacking TAAR1. Specifically, we found NMDA receptor-mediated glutamate transmission deficiency in the PFC, likely leading to the aberrant perseverative and impulsive behaviors that are observed in the absence of TAAR1. Thus, TAAR1-based drugs may provide a novel approach for therapeutic management of psychiatric disorders involving aberrant cortical function.

\section{FUNDING AND DISCLOSURE}

Dr Gainetdinov's work has been partially supported by the research award from F. Hoffmann La-Roche, Basel, Switzerland and by the Russian Science Foundation (project N14-25-00065). Dr Hoener and Dr. Harmeier are employees of F. Hoffmann La-Roche, Basel, Switzerland. The other authors declare no potential conflict of interest.

\section{REFERENCES}

Achat-Mendes C, Lynch LJ, Sullivan KA, Vallender EJ, Miller GM (2012). Augmentation of methamphetamine-induced behaviors 
in transgenic mice lacking the trace amine-associated receptor 1. Pharmacol Biochem Behav 101: 201-207.

Bari A, Robbins TW (2013). Inhibition and impulsivity: behavioral and neural basis of response control. Prog Neurobiol 108: 44-79.

Berger DF, Sagvolden T (1998). Sex differences in operant discrimination behaviour in an animal model of attentiondeficit hyperactivity disorder. Behav Brain Res 94: 73-82.

Borowsky B, Adham N, Jones KA, Raddatz R, Artymyshyn R, Ogozalek KL et al (2001). Trace amines: identification of a family of mammalian G protein-coupled receptors. Proc Natl Acad Sci U S A 98: 8966-8971.

Bunzow JR, Sonders MS, Arttamangkul S, Harrison LM, Zhang G, Quigley DI et al (2001). Amphetamine, 3,4-methylenedioxymethamphetamine, lysergic acid diethylamide, and metabolites of the catecholamine neurotransmitters are agonists of a rat trace amine receptor. Mol Pharmacol 60: 1181-1188.

Cisneros IE, Ghorpade A (2014). Methamphetamine and HIV-1induced neurotoxicity: role of trace amine associated receptor 1 cAMP signaling in astrocytes. Neuropharmacology 85: 499-507.

Collingridge GL, Volianskis A, Bannister N, France G, Hanna L, Mercier $M$ et al (2013). The NMDA receptor as a target for cognitive enhancement. Neuropharmacology 64: 13-26.

Coull JT, Cheng RK, Meck WH (2011). Neuroanatomical and neurochemical substrates of timing. Neuropsychopharmacology 36: 3-25.

Dalley JW, Cardinal RN, Robbins TW (2004). Prefrontal executive and cognitive functions in rodents: neural and neurochemical substrates. Neurosci Biobehav Rev 28: 771-784.

Di Cara B, Maggio R, Aloisi G, Rivet JM, Lundius EG, Yoshitake T et al (2011). Genetic deletion of trace amine 1 receptors reveals their role in auto-inhibiting the actions of ecstasy (MDMA). J Neurosci 31: 16928-16940.

Espinoza S, Gainetdinov RR (2014). Neuronal functions and emerging pharmacology of TAAR1. Top Med Chem (e-pub ahead of print 9 December 2014; doi: 10.1007/7355_2014_78).

Espinoza S, Salahpour A, Masri B, Sotnikova TD, Messa M, Barak LS et al (2011). Functional interaction between trace amine-associated receptor 1 and dopamine D2 receptor. Mol Pharmacol 80: 416-425.

Floresco SB, Zhang Y, Enomoto T (2009). Neural circuits subserving behavioral flexibility and their relevance to schizophrenia. Behav Brain Res 204: 396-409.

Fumagalli F, Calabrese F, Luoni A, Bolis F, Racagni G, Riva MA (2012). Modulation of BDNF expression by repeated treatment with the novel antipsychotic lurasidone under basal condition and in response to acute stress. Int J Neuropsychopharmacol 15: 235-246.

Gardoni F, Di Luca M (2006). New targets for pharmacological intervention in the glutamatergic synapse. Eur J Pharmacol 545: $2-10$.

Kim MJ, Dunah AW, Wang YT, Sheng M (2005). Differential roles of NR2A- and NR2B-containing NMDA receptors in Ras-ERK signaling and AMPA receptor trafficking. Neuron 46: 745-760.

Leo D, Mus L, Espinoza S, Hoener MC, Sotnikova TD, Gainetdinov RR (2014). Taar1-mediated modulation of presynaptic dopaminergic neurotransmission: role of D2 dopamine autoreceptors. Neuropharmacology 81: 283-291.

Lindemann L, Hoener MC (2005). A renaissance in trace amines inspired by a novel GPCR family. Trends Pharmacol Sci 26: 274-281.

Lindemann L, Meyer CA, Jeanneau K, Bradaia A, Ozmen L, Bluethmann $\mathrm{H}$ et al (2008). Trace amine-associated receptor 1 modulates dopaminergic activity. J Pharmacol Exp Ther 324: 948-956.

Lynch LJ, Sullivan KA, Vallender EJ, Rowlett JK, Platt DM, Miller GM (2013). Trace amine associated receptor 1 modulates behavioral effects of ethanol. Subst Abuse 7: 117-126.

Matell MS, Bateson M, Meck WH (2006). Single-trials analyses demonstrate that increases in clock speed contribute to the methamphetamine-induced horizontal shifts in peak-interval timing functions. Psychopharmacology (Berl) 188: 201-212.

Matell MS, Meck WH (2000). Neuropsychological mechanisms of interval timing behavior. Bioessays 22: 94-103.

Matell MS, Portugal GS (2007). Impulsive responding on the peakinterval procedure. Behav Processes 74: 198-208.

Meck WH, Penney TB, Pouthas V (2008). Cortico-striatal representation of time in animals and humans. Curr Opin Neurobiol 18: 145-152.

Millan MJ, Agid Y, Brune M, Bullmore ET, Carter CS, Clayton NS et al (2012). Cognitive dysfunction in psychiatric disorders: characteristics, causes and the quest for improved therapy. Nat Rev Drug Discov 11: 141-168.

Mohn AR, Gainetdinov RR, Caron MG, Koller BH (1999). Mice with reduced NMDA receptor expression display behaviors related to schizophrenia. Cell 98: 427-436.

Pei Y, Lee JA, Leo D, Gainetdinov RR, Hoener MC, Canales JJ (2014). Activation of the trace amine-associated receptor 1 prevents relapse to cocaine seeking. Neuropsychopharmacology 39: 2299-2308.

Revel FG, Moreau JL, Gainetdinov RR, Bradaia A, Sotnikova TD, Mory $\mathrm{R}$ et al (2011). TAAR1 activation modulates monoaminergic neurotransmission, preventing hyperdopaminergic and hypoglutamatergic activity. Proc Natl Acad Sci USA 108: 8485-8490.

Revel FG, Moreau JL, Gainetdinov RR, Ferragud A, VelazquezSanchez C, Sotnikova TD et al (2012). Trace amine-associated receptor 1 partial agonism reveals novel paradigm for neuropsychiatric therapeutics. Biol Psychiatry 72: 934-942.

Revel FG, Moreau JL, Pouzet B, Mory R, Bradaia A, Buchy D et al (2013). A new perspective for schizophrenia: TAAR1 agonists reveal antipsychotic- and antidepressant-like activity, improve cognition and control body weight. Mol Psychiatry 18: 543-556.

Sagvolden T, Jenssen JR, Brorson IW (1983). Rate-dependent effects of methylphenidate (Ritalin) on fixed-interval behavior in rats. Scand J Psychol 24: 231-236.

Seamans JK, Durstewitz D, Christie BR, Stevens CF, Sejnowski TJ (2001). Dopamine D1/D5 receptor modulation of excitatory synaptic inputs to layer $\mathrm{V}$ prefrontal cortex neurons. Proc Natl Acad Sci U S A 98: 301-306.

Sotnikova TD, Caron MG, Gainetdinov RR (2009). Trace amineassociated receptors as emerging therapeutic targets. Mol Pharmacol 76: 229-235.

Sukhanov I, Espinoza S, Yakovlev DS, Hoener MC, Sotnikova TD, Gainetdinov RR (2014). TAAR1-dependent effects of apomorphine in mice. Int J Neuropsychopharmacol 17: 1683-1693.

Thorn DA, Jing L, Qiu Y, Gancarz-Kausch AM, Galuska CM, Dietz $\mathrm{DM}$ et al (2014a). Effects of the trace amine associated receptor 1 agonist RO5263397 on abuse-related effects of cocaine in rats. Neuropsychopharmacology 39: 2309-2316.

Thorn DA, Zhang C, Zhang Y, Li JX (2014b). The trace amine associated receptor 1 agonist RO5263397 attenuates the induction of cocaine behavioral sensitization in rats. Neurosci Lett 566: 67-71.

Tritsch NX, Sabatini BL (2012). Dopaminergic modulation of synaptic transmission in cortex and striatum. Neuron 76: 33-50.

Ultanir SK, Kim JE, Hall BJ, Deerinck T, Ellisman M, Ghosh A (2007). Regulation of spine morphology and spine density by NMDA receptor signaling in vivo. Proc Natl Acad Sci U S A 104: 19553-19558.

Urbach YK, Bode FJ, Nguyen HP, Riess O, von Horsten S (2010). Neurobehavioral tests in rat models of degenerative brain diseases. Methods Mol Biol 597: 333-356.

Wolinsky TD, Swanson CJ, Smith KE, Zhong H, Borowsky B, Seeman $\mathrm{P}$ et al (2007). The Trace Amine 1 receptor knockout mouse: an animal model with relevance to schizophrenia. Genes Brain Behav 6: 628-639.

Supplementary Information accompanies the paper on the Neuropsychopharmacology website (http://www.nature.com/npp) 